\title{
A Retrospective Study on Post Burns Bacterial Infection Spectrum
}

\author{
Dr.T.Srinivasan, M.S,(1)Dr.M.Muralidharan.(2) \\ ${ }^{I}$ (Associate Professor, Department of General Surgery, GovernmentThiruvarur Medical \\ College/TamilnaduDr.M.G.R Medical University, India). \\ ${ }_{2}^{2}$ (Post Graduate in M.S. General Surgery, Department of General Surgery, Coimbatore Medical \\ College/TamilnaduDr.M.G.R Medical University, India)
}

\begin{abstract}
:
Background: Burn wound infections diagnosis and treating them appropriate antibiotics can prevent bacteremia, sepsis or multiple organ dysfunction syndrome. Also early detection of organisms can prevent delayed healing and also decrease the post burns contracture.Burn wound cellulitis, and invasive burn wound infection were included in the study.

Methods: Methicillin Resistant Staphylococus Aureus, Klebsiella, E.Coli were the most common Microorganisms Detected. The study was conducted on fifty patients for a period of six months form June 2016 to December 2016 at Coimbatore Medical College Coimbatore.

Results: KlebsiellaPnuemonia was found to be the most common bacterial organism, and found to be sensitive to Cefeperazon+Sulbactum.

Conclusion: New Antibiotic Policy to be formed for Burns Ward Patient as the routine antibiotic are found to be resistant to these organisms.
\end{abstract}

Keywords: Burns Wound Infection, Cefaperozone, Klebsiella Pneumonia, Sulbactum

\section{Primary Objectives}

\section{Objectives}

To analyze the common bacteria organism causing infection in post burn patient at burns ward.

Secondary objectives:

To analyze culture and sensitivity pattern of common bacteria in post burn infection,

To modify/evaluate existing antibiotic policy.

\section{Burn Wound Infection}

An ability to make the diagnosis of burn wound infection is important. A clinically focused set of burn wound infection definitions has recently been published and is summarized as follows.

\section{Review Of Literature}

\section{Burn Impetigo}

Diagnostic points - Loss of epithelium from previously epithelialized surface; not related to local trauma.

Treatment strategies - Regular cleaning of debris and exudates; topical antistaphylcoccal antibiotics; grafting of chronically unstable areas of epithelium.

\section{Burn-Related Surgical Wound Infection}

Diagnostic points - Infection is surgically created would that has not yet epithelialized; includes loss of any overlying graft or membrane

Treatment strategies - Regular cleaning of debris and exudates; systemic and topical antistaphylococcal antibiotics; grafting of chronically unstable areas of epithelium

\section{Burn Wound Cellulitis}

Diagnostic points - Infection occurs in uninjured skin surrounding a wound; signs of local infection progress beyond what is expected from burn-related inflammation

Treatment strategies - Systemic antibiotics directed against Streptococcus pyogenes; proper treatment of primary wound

\section{Invasive Burn Wound Infection}

Diagnostic points - Infection occurs in unexcised burn and invades viable underlying tissue; diagnosis may be supported by results from histologic examination or quantitative culture 
Treatment strategies - Systemic antibiotics directed against presumed pathogen; wound excision, with biologic closure when possibleTwo of these, burn wound cellulitis and invasive burn wound infection, are seen with some regularity by clinicians outside a burn center environment. Burn wound cellulitis usually manifests with progressive erythema, swelling, and pain in the uninjured skin around a wound. Usually, this is seen in the first few days after the burn occurred and is typically caused by $\mathrm{S}$ pyogenes. Infection can progress rapidly, but it is generally sensitive to penicillin. Excision of associated deep eschar can be essential to the successful treatment of cellulitis. Elevation to reduce edema is an important adjunt.Initial evaluation and management of the burn patient. Burn wound cellulitis manifests with increasing erythema, swelling, and pain in uninjured skin around the periphery of a wound. Invasive burn wound infection is a rapid proliferation of bacteria in burn eschar that invades underlying viable tissues. A change in color, new drainage, and, occasionally, a foul or sickly sweet odor are clinical findings. Pseudomonal and other gram-negative species are common causes. This infection can be life-threatening and usually requires combined treatment with surgery and antibiotics. Initial evaluation and management of the burn patient. Invasive burn wound infection implies that bacteria or fungi are proliferating in eschar and invading underlying viabletissues. These wounds display a change incolor, new drainage, and often a foul odor. These infections are life-threatening.

\section{Burns Infections}

Burn wound infection is problematic because it delays healing, encourages scarring and may result in bacteremia, sepsis or multiple-organ dysfunction syndrome (a.k.a. organ failure) whereby organs from several systems are unable to maintain homeostasis on their own requiring immediate medical attention.Bacteria and fungi are the most common pathogens of burn wounds. These microns form multi-speciesbiofilms on burn wounds within 48-72 hours of injury. Organisms originate from the patient's own skin, gut and respiratory flora, as well as from contact with contaminated health care environments and workers. Gram-positive bacteria are some of the first to colonize burns, followed quickly by gram-negative. Fungal infection tends to occur in the later stages after the majority of bacteria have been eliminated by topical antibiotics. Two bacterial species, methicillin-resistant Staphylococcus aureus (MRSA) and Pseudomonas areuginosas will be examined in depth in this page as they are two of the most prevalent infective agents. These two species have proven particularly difficult to treat because they posses a large number of virulence factors and antimicrobial resistance genes.

\section{Methicillin- resistant Staphylococcus aureus}

MRSA are gram-positive, spherical microbes and some of the earliest colonizers of burn wounds. These bacteria possess capabilities for both aerobic and anaerobic metabolism. Their genome is contained on one circular chromosome, with antibiotic resistance encoded in transposons. MRSA dwell in the sweat glands, hair follicles and mucous membranes of humans. MRSA can be difficult to eradicate because they often can colonize a host for a long time before causing an infection; until symptoms of infection emerge, MRSA remains undetected and untreated.

Staphylococcus aureus resistance to methicillin and other similar antibiotics is due to the altered structure of penicillin binding proteins. This mutation is caused by resistance genes that are carried in the staphylococcal cassette chromosome (SCC) mec, a mobile genetic element. The cassette encodes for an insertion sequence element, recombinases and regulatory genes. Five versions of SCCmec have been identified, each of which confers resistance to slightly different sets of agents.

Besides those that confer antibiotic resistance, MRSA virulence factors include adhesion proteins, colonization proteins, superantigens, exotoxins, and pore-forming toxins. Adhesion proteins allow MRSA to adhere exceptionally well to host tissues, which makes it difficult for the body to clear the pathogens. Colonization proteins help the bacteria to compete more effectively with other bacteria, both within their own species and with others. Some of these proteins also increase salt tolerance which could beneficial in instances of infection where low perfusion results in high external osmolarity, such as in burn wounds. Superantigens instigate an elevated immune response in the body, sometimes up to $20 \%$ greater than would be triggered by bacteria not in possession of this virulence factor. This is particularly dangerous for patients because it can cause high fevers, shock, multiple organ failure and/or toxic shock syndrome. Pore-forming toxins can lead to vascular leakage, hypovolemic shock and tissue necrosis. It is for these reasons that MRSA infections are so dangerous to patients whom are already immune-compromised.

\section{Pseudomonas aeruginosa}

P.aeruginosa are the most common source of burn infections. They are gram-negative bacilli that possess a single supercoiled circular chromosome. P. aeruginosa are facultative aerobes capable of fermentation that live in the human gut. These bacteria are known for their tendency to cause disease in immunecompromised patients, such as those with AIDS or cystic fibrosis, but rarely in healthy individuals. 
P. aeruginosa use quorum sensing to induce the production of virulence factors such as proteases, hemolysins, exotoxin A andpyocyanin.

These bacteria posses two quorum-sensing systems: one that regulates proteases and another that regulates hemolysins. Though separate systems, the two interact through products of the protease system controlling the hemolysin system at both transcriptional and posttranslational levels. While it has been widely held that quorum sensing is required for biofilm formation, new research has been put forth that challenges that position. A team of scientists out of Auburn University investigated the development of P. aeruginosa, one wild type (WT) and the other quorum-sensing deficient (QS). Eschar samples were examined using fluorescence and scanning electron microscopy at 8,24 and $48 \mathrm{~h}$ after infection. Both strains developed biofilms at the same pace, although WT biofilms were somewhat denser than the QS strain. It had been previously demonstrated that the WT strain was more virulent than the QS. This study suggests that the difference in virulence is not due to impaired biofilms formation, rather it is likely due to differences in the expression of virulence factors. It seems that quorum-sensing knockout strains lost some other genes in the process of mutation.

Proteases are virulence factors that degrade the integrity of the host's physical barriers by splitting proteins and amino acids, allowing for deeper infiltration of infection. Exotoxin A halts the synthesis of proteins, causing local tissue damage, immunosuppression and cell death. Hemolysins act like detergents to break down lipids in epithelial cells allowing, like the proteases, the bacilli to penetrate the host resulting in the spread of infection. In addition, genes encoding proteins for pili and flagella are also important to P. aeruginosa since without these structures, bacteria are unable to navigate their environment and form biofilms.

P. aeruginosa infections are difficult to treat because these microbes possess multiple strategies for eluding predators, including multidrug efflux pumps, antibiotic-modifying enzymes, ad tough other membranes with low permeability.

\section{Methodology: \\ Study type:}

Retrospective study.

\section{Sample size:}

45 patients.

Study period:

October 2016 to December 2016, at burns ward, department of gen. surgery in Coimbatore medical college hospital.

\section{Study method:}

Criteria:

Pus for culture and sensitivity taken from post burn infection site.

\section{Inclusion criteria:}

All patients in burns ward with features of infection at burn site.

\section{Exclusion criteria:}

Immune-compromised patients.

\section{Sex distribution:}

\section{Data Analysis}

\begin{tabular}{|l|l|}
\hline MALE & FEMALE \\
\hline 15 & 30 \\
\hline
\end{tabular}




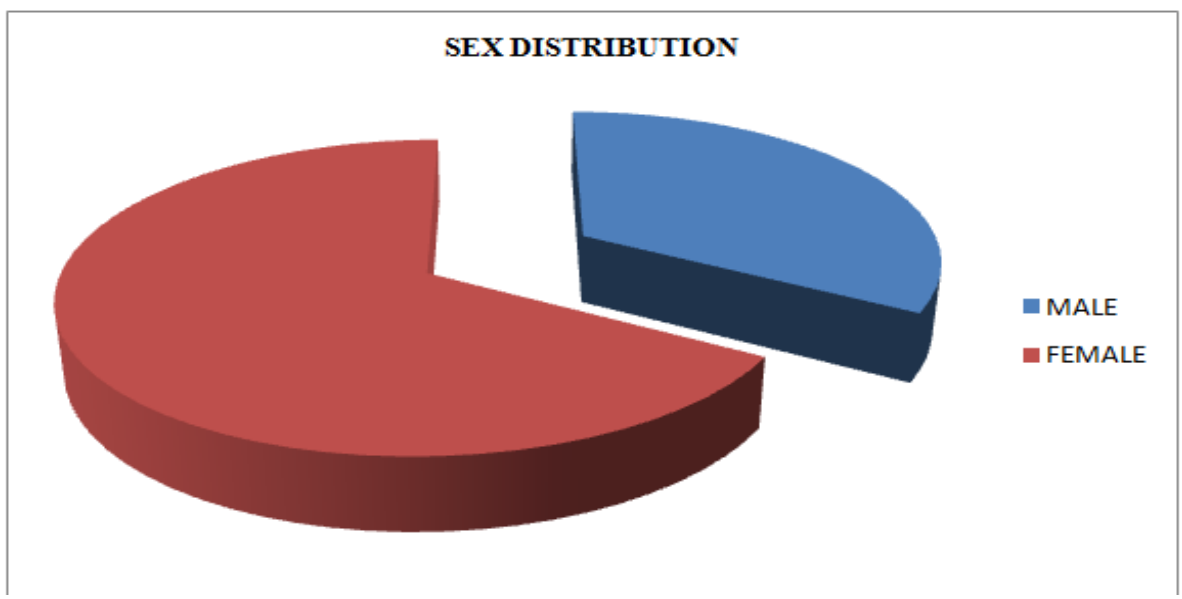

Bacterial spectrum:

\begin{tabular}{|l|l|l|l|}
\hline Klebsiella & Escherichia Coli & $\begin{array}{l}\text { Staphylococcus } \\
\text { Aureus }\end{array}$ & Others \\
\hline 30 & 9 & 4 & 2 \\
\hline
\end{tabular}

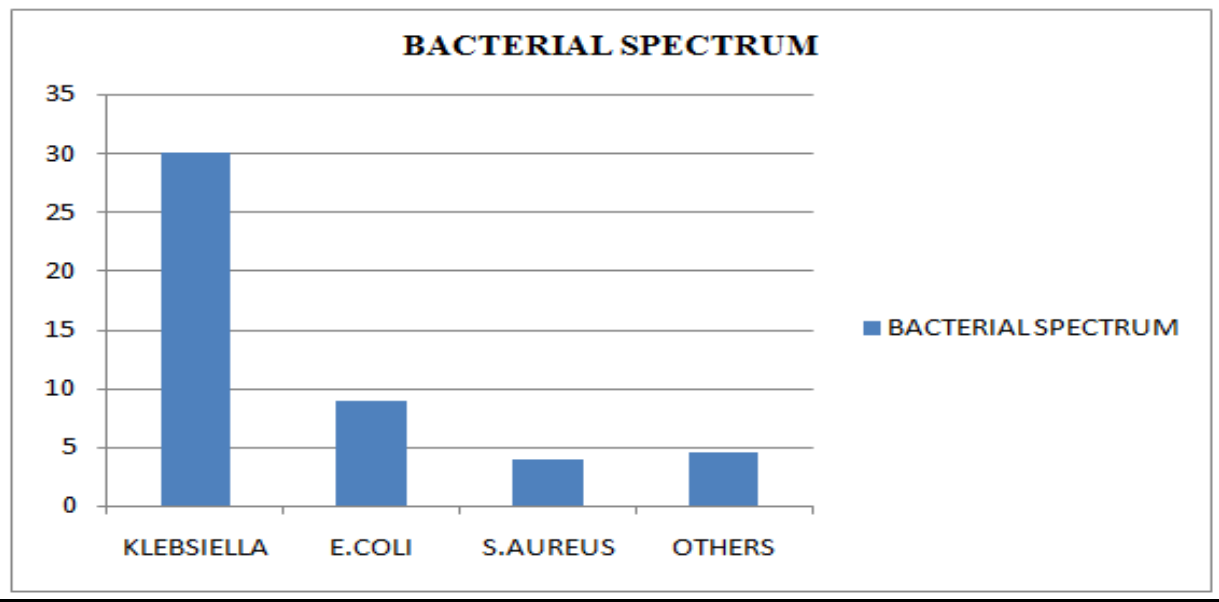

Sensitivity pattern:

\begin{tabular}{|l|l|l|}
\hline Cefoperazone Sulbactem & Ofloxacin & Amikacin \\
\hline $40 / 45$ & $27 / 45$ & $33 / 45$ \\
\hline
\end{tabular}

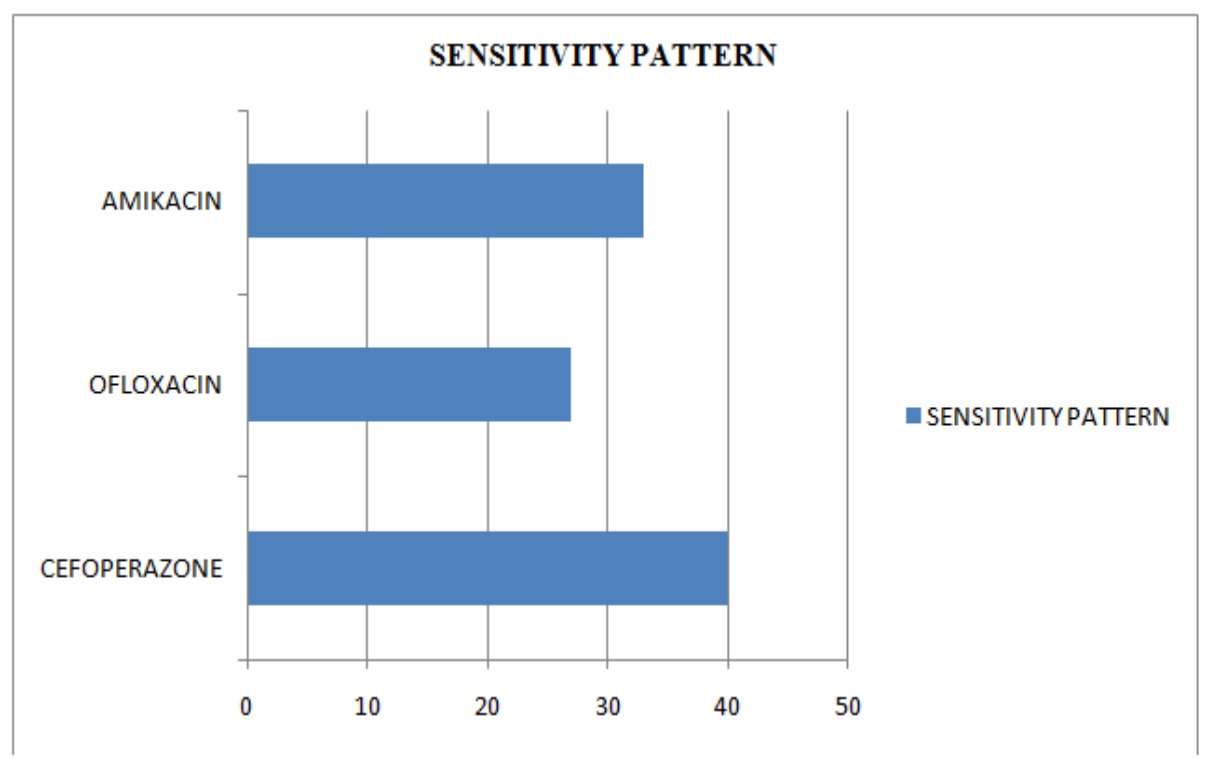




\section{Conclusion}

1. Common bacterial organism in post burns infection was Klebsiella pneumonia.

2. Most of the organisms were sensitive to cefaperazone+sulbactum, ofloxacin and amikacin.

3. Most of the antibiotics included as first and second line drugs in our empirical antibiotic policy was found to be resistant to these organisms. Hence a separate and new elaborate antibiotic policy exclusively for burns ward should be formulated.

\section{References}

[1]. Atiyeh, B.S., Costagliola, M. Hayek, S.H., Dibo.,Dibo, S.A (2007). Effect of silver on burn wound infectio control and healing: Review of the literature. Burns 33, 139-148.

[2]. "Burn incidence and treatment in the U.S.: 2007 Fact sheet." (2005). American Burn Association. http://www.ameriburn.org/resources factsheet php (Accessed 14 apr 2009)

[3]. Church, D., Elsayed, S., Reid, O., Winston, B., Lindsay, R. (2006) Burn Wound Infections. Cliinical Microbiology Reviews, 19(20, 403-434.

[4]. Lyczak, J.b., Cannon, C.1., Pier, G.B. (2000). Establishmet of Pseudomonas aeruginosa infection: lessons from a versatile opportunist.Microbes and Infection 2, 1051-1060.

[5]. McVay, C.S., Velasquez, M., Fralick, J.A.(2007). Phage therapyof Pseudomonas aeruginosa infection in a mouse burn wound model. Antimicrobial Agents and Chemotherapy 51(6), 1934-1938.

[6]. Merabishvili, M., Pirnay, J., Verbeken, G., Chanshvili, N., Tediashivili, M., Lashkhi, N., Glonti, T., Kroylov, V., Mast, J., Van Parys, L., Lavigne, R., Volcaert, G., Mattheus, W., Verween, G., De Corte, P., rose, T., Jennes, S., Zizi, M., De Vos, D., Vaneechoutte, M(2009). Quality-Controlled Small-Scale Production of a Well-defined Bacteriophage Cocktail for Use in Human Clinical Trials. PLoS ONE 4(3): e4944

[7]. Molan, P.C. (2001). Potential of honey in the treatment of wounds and burns. Am J CinDermatol 2(1), 13-19. 